\title{
EDITORIAL
}

\section{Antithrombotics in pulmonary hypertension: more work needed before we turn to newer agents!}

\author{
Laurent Bertoletti*,\#,ף, Xavier Delavenne ${ }^{\#, \uparrow_{,},+}$and David Montani $i^{\S, f, * *}$
}

$\mathbf{T}$ herapeutic research is particularly dynamic in the setting of pulmonary arterial hypertension (PAH). Despite the low prevalence of this rare disease, the efficacy and safety of targeted PAH therapies have been evaluated in randomised controlled trials, providing important data for clinicians, health authorities and patients. Beside these well-studied targeted therapies, "supportive therapy" is also recommended. This includes antithrombotic therapy with vitamin-K antagonists (VKAs) [1]. The background for the use of these agents is principally supported by findings in histopathological and biological studies [2, 3], as well as their potential indirect clinical consequences which may present as perfusion defects evaluated by ventilation/perfusion $\left(V^{\prime} / Q^{\prime}\right)$ scan [4]. Clinical studies evaluating VKAs in the setting of $\mathrm{PAH}$ are, however, lacking. In the prospective study published by Rich et al. [5], VKA use improved prognosis, but the allocation to VKA treatment followed the demonstration of perfusion defects on $V^{\prime} / Q^{\prime}$ scan, and not from proper randomisation. This explains the low level of the related recommendation in the guidelines from the European Respiratory Society and European Society of Cardiology [1], that being a class IIa recommendation for idiopathic or heritable PAH and for PAH due to anorexigens, and an even lower level of recommendation in patients with associated PAH. By extension, anticoagulant therapy has been proposed in other groups of pulmonary hypertension (groups 2,3 or 5) despite the absence of specific studies. Paradoxically, whereas only half of patients with a certain indication for VKA therapy (for example, atrial fibrillation) receive VKA [6], most PAH patients are anticoagulated with VKA $(90 \%$ of the patients included in the French registry) [7]. Conversely, antithrombotic therapy with VKA is the cornerstone of medical therapy in the case of chronic thromboembolic pulmonary hypertension $(\mathrm{CTEPH})$ [1]. However, the main expected goal of VKA in this situation is to prevent a potentially fatal recurrent pulmonary embolism. In addition, VKA may inhibit part of the in situ thrombosis process involved in microvascular disease $[8,9]$ but

\footnotetext{
*Thrombosis Research Group, EA3065, Université Saint-Etienne, Jean Monnet, Saint-Etienne, \#INSERM, CIE3, Saint-Etienne, "Dept of Therapeutic Medicine, CHU Saint-Etienne, Hôpital Nord, Saint-Etienne, 'Laboratory of Pharmacology and Toxicology, CHU Saint-Etienne, Saint-Etienne, ${ }^{\S}$ Université Paris-Sud, Le Kremlin-Bicêtre, ${ }^{f}$ AP-HP, Service de Pneumologie, DHU Thorax Innovation, Hôpital Bicêtre, Le Kremlin-Bicêtre, and **INSERM U999, LabEx LERMIT, Centre Chirurgical Marie Lannelongue, Le Plessis Robinson, France.
}

CORRESPONDENCE: L. Bertoletti, Université de Saint-Etienne, Jean Monnet, Batiment de la Recherche, Saint-Etienne, F-42023, France. E-mail: laurent.bertoletti@gmail.com it does not preclude the deterioration of CTEPH patients [10]. Indeed, recent studies not only found a resistance to fibrinolysis in CTEPH but also in PAH patients [11]. This physiological abnormality should explain, at least in part, the negative results of antiplatelet therapy in $\mathrm{PAH}$ patients [12] and reassure us in our use of anticoagulant therapy, even though we must keep in mind potential safety issues associated with this strategy, such as bleeding.

In this issue of European Respiratory Journal, HeNKENS et al. [13] present a retrospective single centre study providing interesting data on major bleeding in different groups of pulmonary hypertension patients undergoing VKA treatment. The authors analysed bleeding events in 99 patients with idiopathic PAH, 39 patients with $\mathrm{PAH}$ associated with connective tissue disease (CTD-PAH) and 60 CTEPH patients, who received VKA monitored in specialised anticoagulation clinics. In this series, major bleeding (defined in accordance with international guidelines [14]) ranged from 2.4 per 100 patient-years for CTEPH and 5.4 per 100 patient-years for idiopathic $\mathrm{PAH}$, to 19 per 100 patient-years for CTD-PAH. The two main types of major bleeding were gastrointestinal bleeding and haemoptysis. There was one death that was directly related to bleeding, in a patient with a questionable indication for anticoagulation (continuation of VKA therapy 3 months after heart and lung transplantation). Only one other patient suffered from a major bleed involving the central nervous system. It is noteworthy that the use of the tyrosine kinase inhibitor imatinib in the treatment of $\mathrm{PAH}$ may be associated with a high risk of subdural haematoma, particularly in patients receiving anticoagulation therapy [15]. Indeed, the risk:benefit ratio of VKA therapy in the setting of PAH should be discussed according to the subgroup of pulmonary hypertension and the concomitant therapy.

The authors have to be thanked for providing these data focusing on an under-evaluated dimension of such a rare disease, but clinicians should keep in mind some points regarding the results. Because of the retrospective design, the results might be influenced by several types of bias, such as selection or evaluation bias [16]. It should be noted that the population studied was young (for example, about 10 years younger than patients in the French registry for idiopathic $\mathrm{PAH}$ [7] and younger than those in the non-operable group of an international registry for CTEPH [17]). This might contribute to the lower absolute rate of major bleeding compared to patients receiving VKA for secondary prevention of venous thromboembolism in the RIETE registry [18]. It should finally 
explain the very low rate of major bleeding in CTEPH patients, despite these patients being usually older and with many more comorbidities [19], factors usually associated with an increased risk of bleeding. One can hypothesise that CTEPH patients who were not well equilibrated under VKA might not have survived sufficiently long to be evaluated in the referral centre and then in the study [16]. Moreover, a target international normalised ratio (INR) above 2.5 was expected for the majority of patients: for $79 \%$ of idiopathic PAH, $91 \%$ of CTD-PAH and CTEPH. As the risk of bleeding increases significantly with INR, and usually outweighs the benefit of VKA when the INR is above three [20], this unusually high target might severely increase the risk of bleeding.

Then, how should this study be integrated into our clinical practice and in our reflection for future research? First, this study may help us to define the population with a higher risk of major bleeding. Once again, CTD-PAH seems to be associated with the worst outcomes under VKA, which could be explained by the gastrointestinal lesions associated with CTD. Moreover, patients receiving prostacyclins should be followed with caution because of its antiplatelet effects, particularly if they also have thrombocytopenia. In the context of haemoptysis complicating PAH, bronchial artery embolisation remains the treatment of choice and withdrawal of VKA should be proposed in absence of formal indication [21]. Secondly, evaluation of antithrombotic therapy modalities should be discussed on an individual basis, with clinicians experimenting in the usual field of antithrombotic prescription. There are currently very few validated indications of association for VKA and antiplatelet therapy, as for VKA with a target-INR above three [1]. Hence, the combination of antiplatelet therapy and VKA should be solely reserved for some very specific indications, such as a recent arterial stenting in a patient with another indication for VKA (e.g. atrial fibrillation associated with mitral regurgitation as was observed in benfluorex exposed patients [22]). Finally, it seems far too soon to switch PAH patients undergoing VKA therapy to new oral anticoagulants. The field of antithrombotic therapy is undergoing a complete revolution in the setting of non-valvular atrial fibrillation and venous thromboembolism initial therapy. Dabigatran [23] and rivaroxaban [24] have demonstrated their efficacy and safety in both conditions, while the results of apixaban in atrial fibrillation [25] have aroused huge hopes in venous thromboembolism. In the context of atrial fibrillation, all of them have demonstrated a lower risk of major bleeding versus VKA without any need for dosage monitoring. However, their use in the setting of pulmonary hypertension cannot be promoted currently. The first reason is obvious: these drugs have not been evaluated in the setting of pulmonary hypertension and the management of bleeding under these drugs has not even been resolved; in addition the effects of these agents are difficult to reverse in emergency situations. The second reason is the increase in the risk of gastrointestinal bleeding found (versus VKA) in the main phase-III atrial fibrillation trials for dabigatran and rivaroxaban (gastrointestinal bleeding was also the most frequent bleeding localisation in the study of HENKENS et al. [13]). The last and maybe more important issue is related to the pharmacological properties of these new oral anticoagulants. Their absorption and metabolism is highly modified by transporters and hepatic cytochrome, which may be modified by targeted PAH therapy [26]. The monitoring of their biological concentration remains difficult [27] and is under debate [28].

In conclusion, the benefit/risk ratio of VKA therapy is still difficult to evaluate in the setting of pulmonary hypertension and the subgroup of pulmonary hypertension and the associated comorbidities should be considered. The development of new oral anticoagulants raises potential interest in the field of $\mathrm{PAH}$; however, it would be premature to consider these treatments as an option in the absence of specific experimental and pharmacological studies.

\section{STATEMENT OF INTEREST}

Conflict of interest information can be found alongside the online version of this article at www.erj.ersjournals.com

\section{ACKNOWLEDGEMENTS}

The authors thank Carolina Sanz-Hawkins (Dept of Clinical Research, S\&H Medical Science Service, Madrid, Spain) and Laura C. Price (Dept of Pulmonary Hypertension, National Heart and Lung Institute, Imperial College London, Royal Brompton Hospital, London, UK), for English correction and critical review.

\section{REFERENCES}

1 Galiè N, Hoeper MM, Humbert M, et al. Guidelines for the diagnosis and treatment of pulmonary hypertension. Eur Respir J 2009; 34: 1219-1263.

2 Fuster V, Steele PM, Edwards WD, et al. Primary pulmonary hypertension: natural history and the importance of thrombosis. Circulation 1984; 70: 580-587.

3 Johnson SR, Granton JT, Mehta S. Thrombotic arteriopathy and anticoagulation in pulmonary hypertension. Chest 2006; 130: 545-552.

4 Seferian A, Helal B, Jaïs X, et al. Ventilation/perfusion lung scan in pulmonary veno-occlusive disease. Eur Respir J 2012; 40: 75-83.

5 Rich S, Kaufmann E, Levy PS. The effect of high doses of calciumchannel blockers on survival in primary pulmonary hypertension. N Engl J Med 1992; 327: 76-81.

6 Eikelboom JW, Weitz JI. Another oral thrombin inhibitor for stroke prevention in atrial fibrillation? Thromb Haemost 2010; 103: 481-483.

7 Humbert M, Sitbon O, Chaouat A, et al. Survival in patients with idiopathic, familial, and anorexigen-associated pulmonary arterial hypertension in the modern management era. Circulation 2010; 122: 156-163.

8 Wynants M, Quarck R, Ronisz A, et al. Effects of C-reactive protein on human pulmonary vascular cells in chronic thromboembolic pulmonary hypertension. Eur Respir J 2012; 40: 886-894.

9 Delcroix M, Vonk-Noordegraaf A, Fadel E, et al. Vascular and right ventricular remodeling in chronic thromboembolic pulmonary hypertension. Eur Respir J 2013; 41: 224-232.

10 Jenkins D, Mayer E, Screaton N, et al. State-of-the-art chronic thromboembolic pulmonary hypertension diagnosis and management. Eur Respir Rev 2012; 21: 32-39.

11 Miniati M, Fiorillo C, Becatti M, et al. Fibrin resistance to lysis in patients with pulmonary hypertension other than thromboembolic. Am J Respir Crit Care Med 2010; 181: 992-996.

12 Kawut SM, Bagiella E, Lederer DJ, et al. Randomized clinical trial of aspirin and simvastatin for pulmonary arterial hypertension: ASA-STAT. Circulation 2011; 123: 2985-2993.

13 Henkens IR, Hazenoot T, Boonstra A, et al. Major bleeding with vitamin $\mathrm{K}$ antagonist anticoagulants in pulmonary hypertension. Eur Respir J 2013; 41: 872-878.

14 Schulman S, Kearon C. Definition of major bleeding in clinical investigations of antihemostatic medicinal products in nonsurgical patients. J Thromb Haemost 2005; 3: 692-694. 
15 Hoeper MM, Barst R, Galie N, et al. Long-term safety and efficacy of imatinib in pulmonary arterial hypertension. Eur Respir J 2012; 40: Suppl. 56, 583s.

16 Miller DP, Gomberg-Maitland M, Humbert M. Survivor bias and risk assessment. Eur Respir J 2012; 40: 530-532.

17 Pepke-Zaba J, Delcroix M, Lang I, et al. Chronic thromboembolic pulmonary hypertension (CTEPH): results from an international prospective registry. Circulation 2011; 124: 1973-1981.

18 Nieto JA, Solano R, Ruiz-Ribó MD, et al. Fatal bleeding in patients receiving anticoagulant therapy for venous thromboembolism: findings from the RIETE registry. J Thromb Haemost 2010; 8: 1216-1222.

19 Hurdman J, Condliffe R, Elliot CA, et al. ASPIRE registry: Assessing the Spectrum of Pulmonary hypertension Identified at a REferral centre. Eur Respir J 2012; 39: 945-955.

20 Eckman MH, Levine HJ, Pauker SG. Effect of laboratory variation in the prothrombin-time ratio on the results of oral anticoagulant therapy. N Engl J Med 1993; 329: 696-702.

21 Delcroix M, Naeije R. Optimising the management of pulmonary arterial hypertension patients: emergency treatments. Eur Respir Rev 2010; 19: 204-211.
22 Savale L, Chaumais MC, Cottin V, et al. Pulmonary hypertension associated with benfluorex exposure. Eur Respir J 2012; 40: 1164-1172.

23 Schulman S, Kearon C, Kakkar AK, et al. Dabigatran versus warfarin in the treatment of acute venous thromboembolism. N Engl J Med 2009; 361: 2342-2352.

24 Büller HR, Prins MH, Lensin AW, et al. Oral rivaroxaban for the treatment of symptomatic pulmonary embolism. N Engl J Med 2012; 366: 1287-1297.

25 Granger CB, Alexander JH, McMurray JJV, et al. Apixaban versus warfarin in patients with atrial fibrillation. $N$ Engl J Med 2011; 365: 981-992.

26 Bertoletti L, Delavenne X, Montani D, et al. Potential pharmacological interactions between oral pulmonary arterial hypertension (PAH) therapies and new oral anticoagulants. Eur Respir J 2012; 40: Suppl. 56, 274s.

27 Delavenne X, Moracchini J, Laporte S, et al. UPLC MS/MS assay for routine quantification of dabigatran - a direct thrombin inhibitor - in human plasma. J Pharm Biomed Anal 2012; 58: 152-156.

28 Mismetti P, Laporte S. New oral antithrombotics: a need for laboratory monitoring. J Thromb Haemost 2010; 8: 621-626. 